\title{
An in vitro validation method for inertial-sensor-to-bone alignment
}

I. Weygers, M. Kok, T. Seel, F. Olsson, H. De Vroey, D. Shah, O. Taylan, H. Hallez, L. Scheys and K. Claeys

\section{Introduction}

One of the major shortcomings in lower limb kinematic estimation using inertial measurement units (IMU) is the misalignment of sensor axes with respect to the underlying anatomical axes. Approaches that aim to identify this sensor-to-bone misalignment often assume a hinge joint model and estimate this dominant axis of rotation [1]. The presence of secondary kinematics in the tibiofemoral joint violates this model assumption. With common validation techniques e.g. an optical motion capture (OMC) reference, it is difficult to distinguish between violation on model assumptions and the effect of soft tissue artefacts (STA). Furthermore, mechanical setup-based validation often simplifies physiological joint biomechanics. Consequently, we present a validation method on a cadaver for inertial-sensor-to-bone alignment that is able to track the underlying bone movement as a reference during dynamic unloaded motion.

\section{Research question}

Can an in vitro validation method provide a kinematic reference for inertial-sensor-to-bone alignment?

\section{Methods}

Following ethical approval by the institutional board (HNO9) a fresh-frozen cadaveric lower limb, disarticulated at the level of the hip was used for the experiment. Retroreflective marker clusters rigidly attached to the femur and tibia before performing a computed tomography aided in registering the coordinate systems of the bones defined using anatomical landmarks on the surface bone models [2] (Fig. 1). Tibia and femur were equipped with IMUs (Mtw Awinda, Xsens) both rigidly attached on the bone and on the skin with Velcro straps. 54 trials were conducted in different movement planes (vertical, horizontal, varying orientation), range of motion $\left(60^{\circ}\right.$ and $\left.110^{\circ}\right)$, movement duration (15s, 30s and 120s) and movement excitation (fast, slow and a mix of consecutive slow and fast periods). A six-camera $\mathrm{OMC}$ system ( $\mathrm{MX}+, 100 \mathrm{~Hz}$; Vicon) was used to capture marker trajectories and inertial measurements synchronously. Misalignment of the IMU sensing axes and their surrounding optical marker clusters were identified following [3].

\section{Results}

The proposed method provides a reference for the validation of inertial-sensor-to-bone alignment from dynamic motion of an in vitro tibiofemoral knee joint. Preliminary validation results indicate that a dominant axis of rotation, identified from (bone-fixed) inertial sensors [1] coincides with the underlying anatomical femur-fixed flexion axis. However, misalignments observed in horizontal femur-fixed plane (mean $10.34^{\circ}$, SD $6.26^{\circ}$ ) are significantly $(p<0.003)$ larger than in the vertical plane (mean $4.44^{\circ}$, SD $\left.6.31^{\circ}\right)$.

\section{Discussion}

In contrast to in vivo OMC-based validation, the proposed method yields a kinematic reference based on anatomical landmarks that are not affected by STA or palpation errors. Secondary tibiofemoral kinematics (tibial rotation and abduction-adduction movements) violates the assumption of a hinge joint model. This might explain the wide spread in IMU-based estimates of the underlying flexion axis. Nevertheless, the proposed validation method can be used for IMU-based tibiofemoral joint models that incorporate secondary kinematics. 


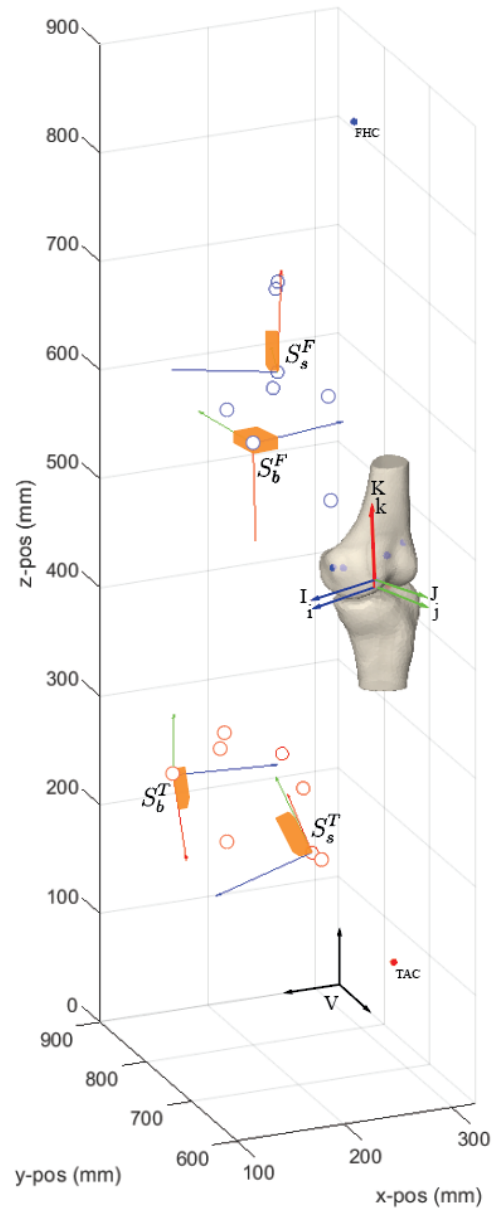

Fig. 1. Measurement set-up: Coordinate systems of Inertial sensors (orange) on the bone (b) and skin (s) and surface bone models.

\section{References}

1. Olsson, F., et al. Joint Axis Estimation for Fast and Slow Movements Using Weighted Gyroscope and Acceleration Constraints. in 2019 22th International Conference on Information Fusion (FUSION). 2019.

2. Grood, E.S. and W.J. Suntay, A joint coordinate system for the clinical description of threedimensional motions: application to the knee. Journal of Biomechanical Engineering, 1983. 105(2): p. 136-44.

3. Lee, J.K. and W.C. Jung, Quaternion-Based Local Frame Alignment between an Inertial Measurement Unit and a Motion Capture System. Sensors (Basel), 2018. 18(11). 